\title{
Constraints on distances to Galactic Centre non-thermal filaments from $\mathrm{HI}$ absorption
}

\begin{abstract}
Subhashis Roy ${ }^{\star}$
National Centre for Radio Astrophysics (TIFR), Pune University Campus, Post Bag No. 3, Ganeshkhind, Pune 411 007, India

Received 25 January 2002 / Accepted 12 February 2003

Abstract. We have studied HI absorption towards three non-thermal filaments (NTFs) Sgr C, G359.54+0.18 and G359.79+0.17 using the Giant Metrewave Radio Telescope (GMRT). Our study, for the first time, constrains the distance of the Sgr C NTF and the HII region seen associated with the NTF in the sky plane, to within a few hundred parsecs from the Galactic Centre (GC). A molecular cloud with a velocity of $-100 \mathrm{~km} \mathrm{~s}^{-1}$ appears to be associated with the central part of the Sgr C NTF. Our study also indicates that the Sgr C HII region is relatively farther away than the NTF along our line of sight, and thereby provides evidence against any possible interaction between the two objects. The NTF G359.54+0.18 shows weak HI absorption $(4 \sigma$ detection) at a velocity of $-140 \mathrm{~km} \mathrm{~s}^{-1}$, which is the velocity of a known dense molecular cloud seen towards the NTF. This cloud is expected to be located within $\sim 200 \mathrm{pc}$ of the GC and thereby provides a lower limit to the distance. The upper limit to the distance of this NTF from the Sun is $10.5 \mathrm{kpc}$. The distance to the NTF G359.79+0.17 is between 5.1 and $10.5 \mathrm{kpc}$ from the Sun.
\end{abstract}

Key words. radio lines: ISM - ISM: clouds - Galaxy: center - ISM: HII regions

\section{Introduction}

The long narrow non-thermal filaments (NTFs) observed in high resolution radio-continuum maps are unique features seen towards only the central $\sim 2^{\circ}$ region of our Galaxy. These structures are less than $1 \mathrm{pc}$ in width, but extend up to $30 \mathrm{pc}$ in length. With the exception of the NTF called the Pelican (Lang et al. 1999), which is nearly parallel to the Galactic plane, all other NTFs are oriented perpendicular to the Galactic plane to within $20^{\circ}$ (Morris \& Serabyn 1996, and references therein). Except for NTF G359.79+0.17, which has a curved morphology reminiscent of a partial shell, all other NTFs have a linear structure. The spectral indices of these structures range from -0.8 to +0.3 (where $\mathrm{S}_{v} \propto v^{\alpha}$ ) (Anantharamaiah et al. 1991) and they are found to be highly polarised at centimetre wavelengths (Morris \& Serabyn 1996). Since these NTFs remain straight despite interaction with nearby molecular clouds, it is believed that the molecular clouds and the NTFs are in pressure equilibrium, which indicates a magnetic field strength of a few milliGauss inside the NTFs (Yusef-Zadeh \& Morris 1987). Magnetic fields of comparable strengths are thought to be present in the central molecular zone (CMZ) located within $\sim 200$ pc of the centre of the Galaxy (Morris \& Serabyn 1996). Before any attempt is made to relate the magnetic field in the NTFs with the processes occurring in the Galactic Centre (GC), it is necessary to establish that these NTFs are actually located in the GC region and are not chance superpositions of foreground or background objects (Lasenby et al. 1989). HI absorption towards the GC "Radio-arc" (Lasenby et al. 1989) and

^ e-mail: roy@ncra.tifr.res.in the "Snake" (Uchida et al. 1992) have indicated that they are located close to the GC, but the distances to the remaining NTFs are not constrained.

An intriguing fact that has been noticed for all the wellstudied NTFs is the interaction between the NTF and molecular clouds (Morris \& Serabyn 1996). It appears that the presence of an HII region near the place of interaction plays a role in the creation and maintenance of the NTFs (Serabyn \& Morris 1994; Staguhn et al. 1998; Uchida \& Guesten 1995). CO observations have indicated the presence of high velocity molecular clouds; $-65 \mathrm{~km} \mathrm{~s}^{-1}$ and $-130 \mathrm{~km} \mathrm{~s}^{-1}$ towards Sgr C (Liszt \& Spiker 1995). It is believed that the HII region located just south of the NTF (known as the Sgr C HII region) is actually embedded in the $-65 \mathrm{~km} \mathrm{~s}^{-1}$ cloud (Liszt \& Spiker 1995; Kramer et al. 1998). Two dense molecular clouds are reported to be associated with the NTF G359.54+0.18. One of the clouds having a velocity of $-140 \mathrm{~km} \mathrm{~s}^{-1}$ is located near the bent portion of the NTF ("E" in Fig. 8). The other cloud with a velocity of $-90 \mathrm{~km} \mathrm{~s}^{-1}$ is located close to the eastern edge of the NTF (Staguhn et al. 1998). However, these reported associations are based on their proximity in the sky plane, and the spatial association of the corresponding objects has yet to be established.

In this paper, we present new $\mathrm{HI}$ absorption measurements towards three NTFs, Sgr C, G359.54+0.18 and G359.79+0.17 made with the Giant Metrewave Radio Telescope (GMRT). These observations not only constrain the distances of these objects, but also test the association of some of the clouds mentioned above with the corresponding NTFs. These three NTFs are located to the south and south west of the Sgr A complex, 
and high resolution radio continuum observations have confirmed the characteristic properties of each of the NTFs: Sgr C (Liszt \& Spiker 1995), G359.54+0.18 (Bally \& Yusef-Zadeh 1989; Yusef-Zadeh et al. 1997) and G359.79+0.17 (Lang \& Anantharamaiah, in preparation).

Due to velocity crowding near Galactic longitude $l=0^{\circ}$, Galactic rotation cannot be used to constrain the distances to these NTFs. Therefore, in this paper, detection of absorption by known HI features has been used to provide constraints on the distances to the NTFs. Since an interferometer resolves the extended HI emission features, no information about the emission features are obtained from our observations. However, absorption by these features against the continuum source can be observed with an interferometer. Therefore, in this section, we briefly summarise the distances and the velocities of the HI features identified from single dish HI emission observations towards the NTFs under study (e.g. Cohen \& Davies 1979), which we will refer to later in the paper. Near the Galactic longitude of $359.5^{\circ}$, two high velocity $\mathrm{HI}$ emission features known as the "Nuclear disk" (Rougoor \& Oort 1960) and the "Molecular ring" (Scoville 1972) have been found. The "Nuclear disk" shows high negative velocity ranging from $\approx-160$ to $-200 \mathrm{~km} \mathrm{~s}^{-1}$, whereas the "Molecular ring" has a velocity of $\approx-135 \mathrm{~km} \mathrm{~s}^{-1}$. Both these features are believed to be nearer than the GC and located at a distance of few hundred parsecs from it (Cohen \& Davies 1979). The emission from the "3 kpc arm" (Rougoor 1964) located at a distance of $\approx 5.1 \mathrm{kpc}$ from the Sun is identified at a velocity near $-53 \mathrm{~km} \mathrm{~s}^{-1}$. At positive velocities, emission near $135 \mathrm{~km} \mathrm{~s}^{-1}$ is seen due to the HI features "XVI" and "I" (Cohen \& Davies 1979), both of which are thought to be located behind the GC. While the feature "XVI" is likely to be located within a few hundred parsecs of the GC (Cohen \& Davies 1979), the feature "I" is thought to be $2 \mathrm{kpc}$ behind the GC (Cohen 1975).

In Sect. 2 of this paper, we discuss the observations and data reduction. The results from our observations are presented in Sect. 3 and their consequences are discussed in Sect. 4. Finally, the conclusions are presented in Sect. 5 .

\section{Observations and data reduction}

The GMRT (Swarup et al. 1991) consists of thirty antennas, distributed over a region of about $25 \mathrm{~km}$, with fourteen of the antennas located within a diameter of about one $\mathrm{km}$ and the remaining arranged in 3 arms each of length $14 \mathrm{~km}$, shaped as an irregular Y. This arrangement provides the necessary $u v$ coverage for mapping both compact and extended sources. The ratio of the longest to the shortest baseline is $\approx 500$ with the shortest projected baseline being $\approx 50$ metres. Table 1 give the details of our observations. All the observations were carried out in the default spectral line mode with 128 frequency channels. A bandwidth of $4 \mathrm{MHz}$ was used to observe G359.54+0.18 and G359.79+0.17 which gave a velocity coverage of $\pm 400 \mathrm{~km} \mathrm{~s}^{-1}$ with a resolution of $6.7 \mathrm{~km} \mathrm{~s}^{-1}$. The Sgr C complex was observed with a bandwidth of $2 \mathrm{MHz}\left( \pm 200 \mathrm{~km} \mathrm{~s}^{-1}\right)$ and $4 \mathrm{MHz}$ $\left( \pm 400 \mathrm{~km} \mathrm{~s}^{-1}\right)$. In all the observations, 3C286 was observed as a primary flux calibrator, 3C287 as the bandpass calibrator and $1748-253$ as the secondary calibrator. The bandpass pattern of the antennas change appreciably as a function of frequency. Therefore, we have not used frequency switching for the bandpass calibration. Instead, we have chosen a bandpass calibrator (3C287) with a high Galactic latitude, so that the effect of Galactic HI absorption on its spectra is less than $1 \%$ (Dickey et al. 1978). During our observations, the automatic measurements of system temperatures were not implemented. However, since in this paper we are not concerned with the absolute value of the flux density, we have applied an approximate correction by scaling the flux of all the fields by the ratio of the estimated flux density (157 mJy) of the small-diameter source G359.87+0.18 (Lazio et al. 1999) at $1.55 \mathrm{GHz}$ to the flux density that we have estimated from our map (ratio $\approx 2.5$ ). All the maps presented in this paper have been corrected for the primary beam pattern of the antennas.

The spectral visibility data were processed using standard NRAO AIPS programs. Bad data due to antenna-based or baseline-based problems were flagged using UVFLG. The data were then calibrated and images of the fields were formed by Fourier inversion and CLEANing (IMAGR). To reduce the effect of comparatively large-scale structures in $\mathrm{HI}$ along the line of sight, we have done a high-pass filtering while making the maps of the three NTFs. Since the NTFs are oriented almost along the east-west direction (i.e. along $u$ ), we have filtered out data having $v \lesssim 2000 \lambda$ and $u \lesssim 4000 \lambda$, because of which any $2-\mathrm{D}$ structure that has a size-scale $\gtrsim 1.5^{\prime}$ will not be visible and linear structures of length $\lesssim 10^{\prime}$ can only be imaged. We have used the AIPS task UVNOU to implement this data filtering in $u$ and $v$. The visibility data were then used to generate the continuum and line images. The continuum map of Sgr C NTF made using the above procedure is shown in Fig. 1. Since the Sgr C HII region is extended, we have applied a lower $u v$ cutoff of $1 \mathrm{k} \lambda$ to the visibility data before making the line and the continuum maps (Fig. 2). During observation of $\mathrm{G} 359.79+0.17$, the GC was located just outside the half power width of the GMRT primary beam. Hence, to minimise the sidelobe response of the GC in the image of the NTF G359.79+0.17, 3-D imaging (available in the recent versions of IMAGR) was used.

Before making the channel maps, the AIPS task UVLSF was used to subtract a constant term across the frequency channels corresponding to the continuum from the visibility data. The GMRT has an FX correlator, for which "Gibbs ringing" due to any sharp feature in the spectrum dies away much faster $\left(\propto \operatorname{sinc}^{2}\right)$ than in a XF correlator. Therefore, we have not applied any spectral smoothing to our data. The variation in the line frequency introduced by the earth's rotation during the observing period is estimated to be much smaller than the frequency channel width and so we have not applied any Doppler corrections to the data. It should be noted here that the RMS noise quoted for each spectrum is the noise estimated from the corresponding images (i.e., from image plane) and are applicable for only $\mid$ velocity| $\gtrsim 20 \mathrm{~km} \mathrm{~s}^{-1}$. For |velocity| $\lesssim 20 \mathrm{~km} \mathrm{~s}^{-1}$, due to emission from the HI gas along the line of sight (velocity crowding occurs when $|l| \approx 0^{\circ}$ ), the system temperature increases and the typical RMS noise is 1.6 times higher than the quoted values. The systematic error in our spectra $\left(\mathrm{e}^{-\tau}\right.$, where $\tau$ is the optical depth) is believed to be less than 0.05 . 
Table 1. Details of our observation.

\begin{tabular}{cccccccccc}
\hline \hline Source name & $\begin{array}{c}\text { RA } \\
(\mathrm{J} 2000)\end{array}$ & $\begin{array}{c}\text { Dec } \\
(\mathrm{J} 2000)\end{array}$ & $\begin{array}{c}l \\
(\mathrm{deg})\end{array}$ & $\begin{array}{c}b \\
(\mathrm{deg})\end{array}$ & $\begin{array}{c}\text { Frequency } \\
(\mathrm{MHz})\end{array}$ & $\begin{array}{c}\text { Band- } \\
\text { width } \\
(\mathrm{MHz})\end{array}$ & Date & $\begin{array}{c}\text { Observing } \\
\text { time (hour) }\end{array}$ & $\begin{array}{c}\text { Anten- } \\
\text { nas }\end{array}$ \\
\hline Sgr C & 174433.8 & -292802 & 359.43 & -0.08 & 1420.4 & 2 & 2000 Oct 06 & 1 & 27 \\
Sgr C & 174433.8 & -292802 & 359.43 & -0.08 & 1420.4 & 2 & 2000 Oct 08 & 2 & 27 \\
Sgr C & 174433.7 & -292800 & 359.43 & -0.08 & 1420.6 & 4 & 2001 May 24 & 2.5 & 28 \\
G359.79+0.17 & 174430.9 & -285950 & 359.82 & 0.17 & 1420.6 & 4 & 2001 May 24 & 3.0 & 26 \\
G359.54+0.18 & 174348.3 & -291126 & 359.57 & 0.20 & 1420.6 & 4 & 2001 May 25 & 3.5 & 28 \\
\hline
\end{tabular}

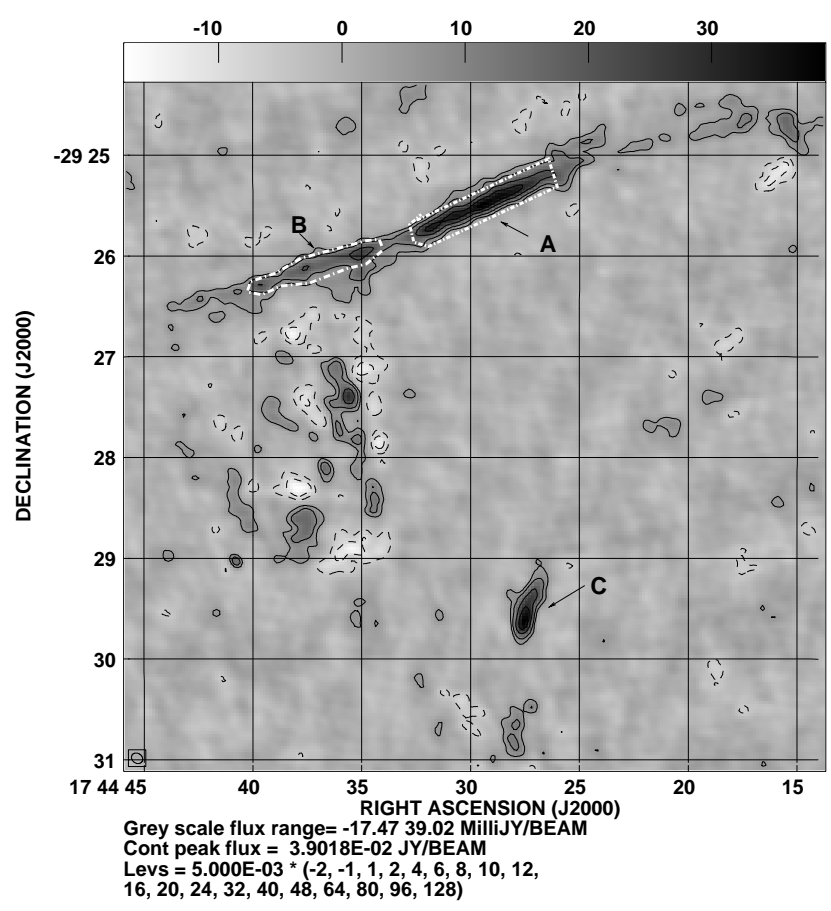

Fig. 1. Continuum map of the Sgr C NTF at $1.4 \mathrm{GHz}$ after high pass filtering. The image has a resolution of $7 \times 6 \operatorname{arcsec}^{2}$, along PA = $56^{\circ}$. The Sgr C HII region is resolved out. RMS noise in the map is $1.6 \mathrm{mJy}$ beam $^{-1}$.

\section{Results}

In this section, we present the absorption spectra towards the target sources and identify the velocity of the HI absorption features. In all the spectra, unless otherwise stated, the $\mathrm{X}$-axis represents the velocity in $\mathrm{km} \mathrm{s}^{-1}$ and Y-axis represents the transmission $\left(I / I_{0}\right)$, where $I$ is the observed flux density of the background source at the given frequency and $I_{0}$ is the actual flux density of the source. We also assume the spin temperature of the atomic hydrogen to be much less than the brightness temperature of the background source and in that case $I / I_{0}=\mathrm{e}^{-\tau}$. All the velocities quoted in this paper are expressed with respect to the local standard of rest and the GC is assumed to be at a distance of $8.5 \mathrm{kpc}$. As is well known, the HI column density is related to its optical depth by the formula, $N_{\mathrm{H}}=1.8 \times 10^{18} \times T_{\mathrm{S}} \times \int \tau \mathrm{d} v$, where, $N_{\mathrm{H}}$ is the column density of the atomic hydrogen, $T_{\mathrm{S}}$ is the spin temperature and $\int \tau \mathrm{d} v$ is

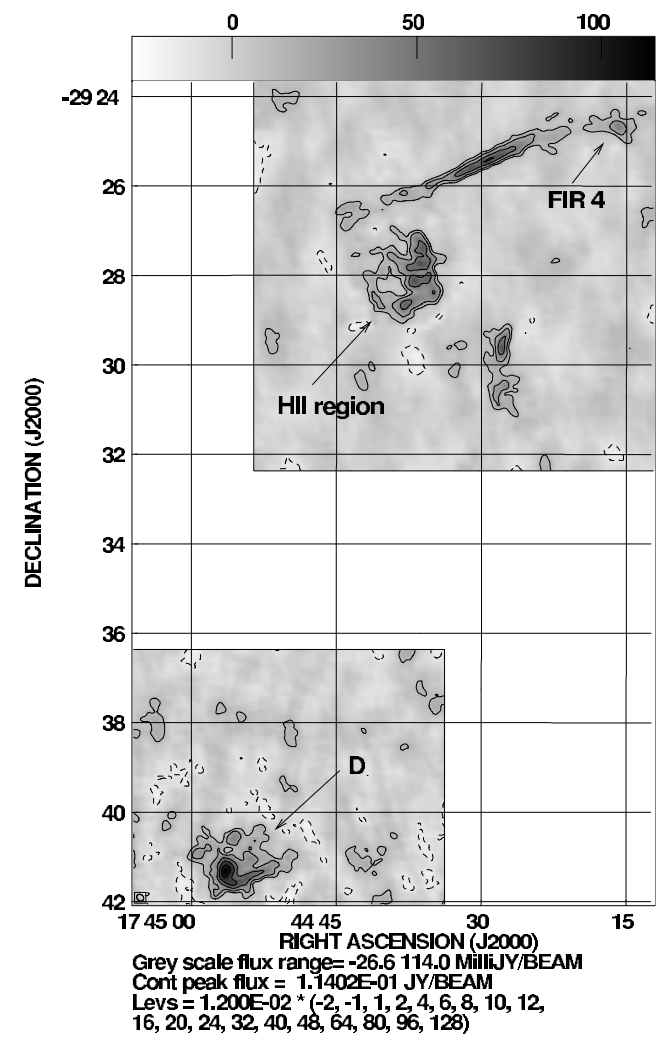

Fig. 2. Continuum map of the $\mathrm{Sgr} \mathrm{C}$ region at $1.4 \mathrm{GHz}$ with a resolution of $10 \times 8 \operatorname{arcsec}^{2}$, along PA $=56^{\circ}$. This is a composite map and shows the Sgr C HII region along with few other sources in the field of view. The rms noise is $4.0 \mathrm{mJy}$ beam.

the velocity integrated optical depth. We use this relation to calculate the HI column density from the observed optical depth.

\section{1. $\operatorname{Sgr} C$}

The absorption spectra towards various parts of the object (Figs. 1 and 2) are shown in Figs. 3 and 4. Figure 3 shows the absorption spectrum towards parts "A" and "B" (see Fig. 1) of the NTF. The spectrum towards part "A" shows several absorption features at negative velocities in addition to the absorption feature at $0 \mathrm{~km} \mathrm{~s}^{-1}$ (line-width $\approx 30 \mathrm{~km} \mathrm{~s}^{-1}$ ). A strong absorption feature near $-54 \mathrm{~km} \mathrm{~s}^{-1}$ is seen due to HI absorption by the " $3 \mathrm{kpc}$ arm". A broad absorption feature is seen between $-100 \mathrm{~km} \mathrm{~s}^{-1}$ and $-200 \mathrm{~km} \mathrm{~s}^{-1}$, with an almost linear 


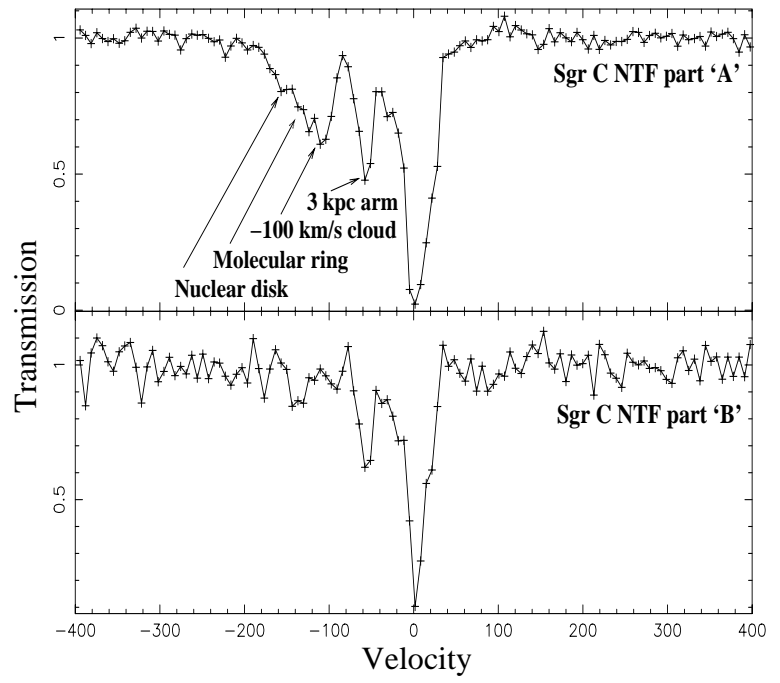

Fig. 3. HI absorption spectrum towards the central bright part and the eastern par (marked "A" and "B" in Fig. 1 respectively) of the Sgr C NTF. The bandwidth is $4 \mathrm{MHz}$. RMS noise in the two spectra is 0.027 and 0.063 respectively.

decrease in optical depth from $\approx 0.5$, at $-100 \mathrm{~km} \mathrm{~s}^{-1}$, to $\approx 0.0$, at $-200 \mathrm{~km} \mathrm{~s}^{-1}$.

The spectrum taken towards part "B" of the NTF (Fig. 3) appears markedly different. The absorption width of the $0 \mathrm{~km} \mathrm{~s}^{-1}$ feature appears to be narrower $\left(\approx 25 \mathrm{~km} \mathrm{~s}^{-1}\right)$ than that observed towards "A". Absorption due to the " $3 \mathrm{kpc}$ arm" is also seen. However, except for a weak $4 \sigma$ absorption feature at $-137 \mathrm{~km} \mathrm{~s}^{-1}$, the wide absorption feature between -100 and $-200 \mathrm{~km} \mathrm{~s}^{-1}$ is not seen.

The absorption spectrum towards the Sgr C HII region with a resolution of $3.3 \mathrm{~km} \mathrm{~s}^{-1}$ is shown in Fig. 4. For comparison, the spectrum of part " $\mathrm{A}$ " having the same velocity resolution is also plotted. Both the spectra show similar absorption features with a few differences, which we note here. Towards the HII region, the broad absorption feature seen between -100 and $-200 \mathrm{~km} \mathrm{~s}^{-1}$ shows at least three main components centred at $-118 \mathrm{~km} \mathrm{~s}^{-1},-138 \mathrm{~km} \mathrm{~s}^{-1}$ and $-175 \mathrm{~km} \mathrm{~s}^{-1}$ with optical depth of $\approx 0.5,0.3$ and 0.2 respectively. The absorption depth at these velocities are similar to what is seen towards part " $\mathrm{A}$ " of the NTF. However, the absorption feature near $0 \mathrm{~km} \mathrm{~s}^{-1}$ is broader towards the HII region (line-width $\approx 33 \mathrm{~km} \mathrm{~s}^{-1}$ ). The HII region shows a feature at $-61.5 \mathrm{~km} \mathrm{~s}^{-1}$, in addition to the absorption by the " $3 \mathrm{kpc}$ arm" observed near $-54 \mathrm{~km} \mathrm{~s}^{-1}$ in both the spectra. However, Part "B" of the NTF being weaker than part "A" in radio continuum, absence of the $-61.5 \mathrm{~km} \mathrm{~s}^{-1}$ feature could not be verified against " $\mathrm{B}$ ".

\subsection{HI absorption spectra towards objects located in the field of Sgr C: sources C, D and FIR-4}

Figure 5 shows the spectra towards source "C" ("3" in Liszt \& Spiker 1995), source "D" and FIR-4 (Fig. 2). The spectrum towards the source " $\mathrm{C}$ " is similar to what is seen towards the Sgr C HII region, but, in place of the wide absorption feature between -100 and $-200 \mathrm{~km} \mathrm{~s}^{-1}$ (Fig. 4), two absorption lines

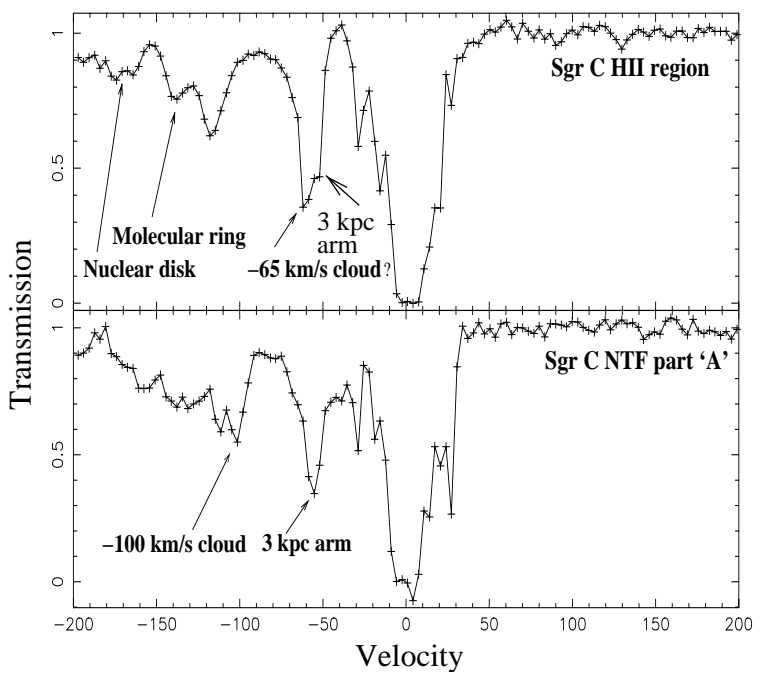

Fig. 4. HI absorption spectrum towards the Sgr C HII region (Fig. 2) and the central bright part (marked "A" in Fig. 1) of the Sgr C NTF. The bandwidth is $2 \mathrm{MHz}$. RMS noise in the two spectra is 0.021 and 0.034 respectively.

are observed at $-123 \mathrm{~km} \mathrm{~s}^{-1}$ and $-170 \mathrm{~km} \mathrm{~s}^{-1}$. Since the continuum emission from the HII region FIR-4 is weak, its absorption spectrum is noisy. However, we identify $\mathrm{HI}$ absorption at $-135 \mathrm{~km} \mathrm{~s}^{-1}$ (5 $\sigma$ detection) and at $-183 \mathrm{~km} \mathrm{~s}^{-1}$ (4 $\sigma$ detection) towards FIR-4. CO emission has been observed towards the source "C" and FIR-4 at $-123 \mathrm{~km} \mathrm{~s}^{-1}$ and $-135 \mathrm{~km} \mathrm{~s}^{-1}$ respectively (Oka et al. 1998). On the other hand, the spectrum towards the southern HII region "D" (Fig. 2), ("1" in Liszt \& Spiker 1995) located just outside the half power width of the GMRT antennas is markedly different. Except the strong absorption near the $0 \mathrm{~km} \mathrm{~s}^{-1}$ (line-width $\approx 30 \mathrm{~km} \mathrm{~s}^{-1}$ ), no other absorption feature could be identified towards this object.

\subsection{NTF G359.79+0.17 and G359.87+0.18}

The continuum image at $20 \mathrm{~cm}$ of the field of NTF G359.79+0.17 is shown in Fig. 6. The absorption spectrum integrated over the NTF is plotted in Fig. 7. The strong absorption near $0 \mathrm{~km} \mathrm{~s}^{-1}$ has a line-width of $\approx 24 \mathrm{~km} \mathrm{~s}^{-1}$. No absorption feature at positive velocities is seen towards this source. However, at negative velocities, an absorption feature can be seen at $-26 \mathrm{~km} \mathrm{~s}^{-1}$, and a weaker feature at $-58 \mathrm{~km} \mathrm{~s}^{-1}$, which coincides with the line of sight velocity of the " $3 \mathrm{kpc}$ arm".

Figure 7 also shows the absorption spectrum towards the extragalactic source G359.87+0.18. Strong absorption near $0 \mathrm{~km} \mathrm{~s}^{-1}$ with a line-width of $\approx 40 \mathrm{~km} \mathrm{~s}^{-1}$, and absorption at $-53 \mathrm{~km} \mathrm{~s}^{-1}$ are observed. Lazio et al. (1999) have observed HI absorption against G359.87+0.18, and the aforementioned features match with their spectrum. However, the present observations have a wider velocity coverage than Lazio et al. (1999) and we detect an additional absorption feature at $+140 \mathrm{~km} \mathrm{~s}^{-1}$. 


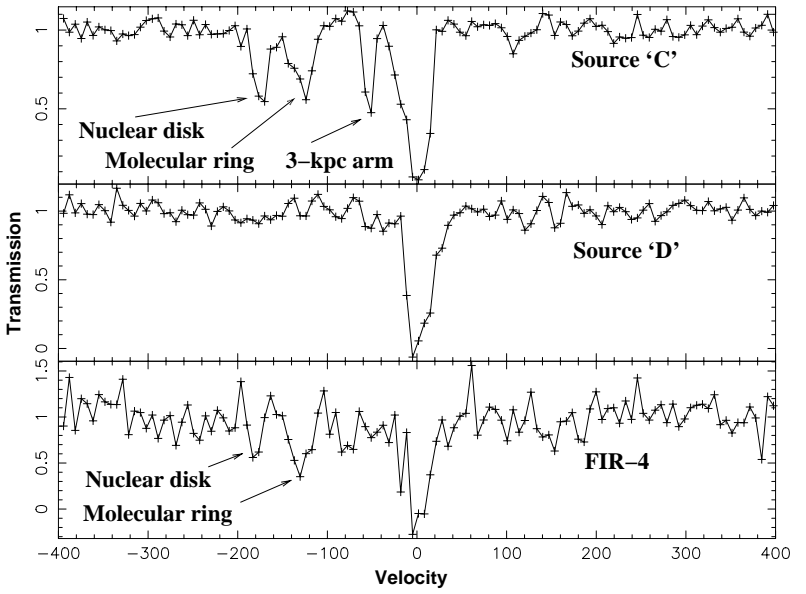

Fig. 5. HI absorption spectrum towards the source "C" (Fig. 1), "D" (Fig. 2) and FIR-4, located in the field of Sgr C. The bandwidth is $4 \mathrm{MHz}$. RMS noise in the three spectra are $0.078,0.05$ and 0.2 respectively.

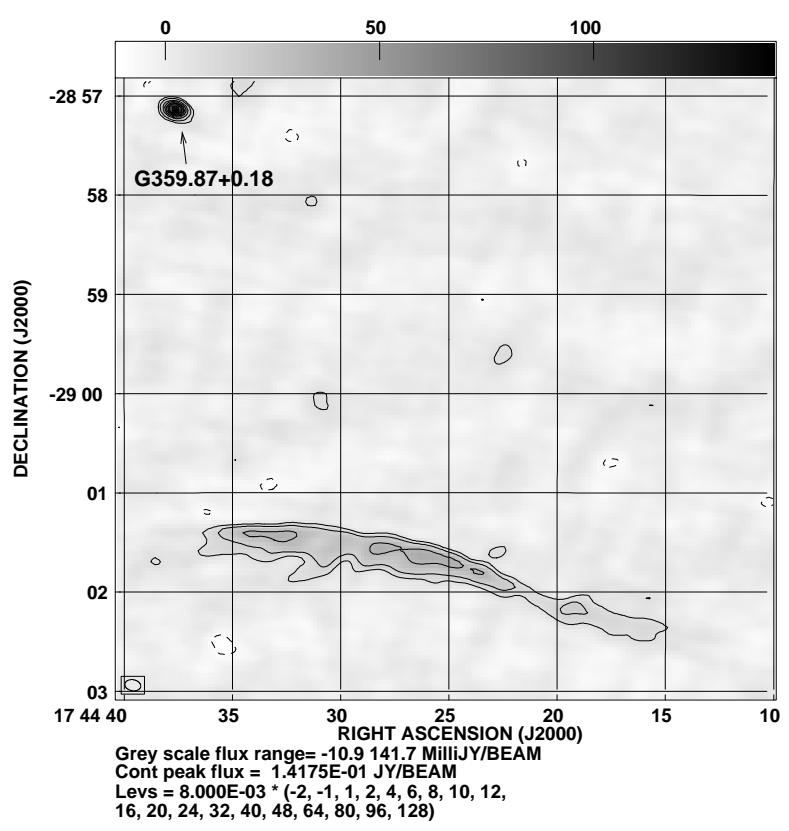

Fig. 6. Continuum image of the NTF G359.79+0.17 at $1.4 \mathrm{GHz}$ with a resolution of $9.7 \times 6.7 \operatorname{arcsec}^{2}$, along PA $=79^{\circ}$. The rms noise is

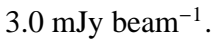

\subsection{NTF G359.54+0.18}

The continuum image of the NTF G359.54+0.18 is shown in Fig. 8 and the absorption spectrum integrated over the NTF is plotted in Fig. 9. The absorption spectrum is quite similar to the one seen towards $\mathrm{G} 359.79+0.17$, with components at $-26 \mathrm{~km} \mathrm{~s}^{-1}$ and $-53 \mathrm{~km} \mathrm{~s}^{-1}$. The strong HI absorption near $0 \mathrm{~km} \mathrm{~s}^{-1}$ has a line-width of $\approx 24 \mathrm{~km} \mathrm{~s}^{-1}$. Staguhn et al. (1998) have found a dense molecular cloud at $-140 \mathrm{~km} \mathrm{~s}^{-1}$ near the bent portion of the NTF (position of the molecular cloud is denoted by "E" in Fig. 8). HI spectrum taken towards this region of the NTF (denoted by "F" in Fig. 8) shows absorption

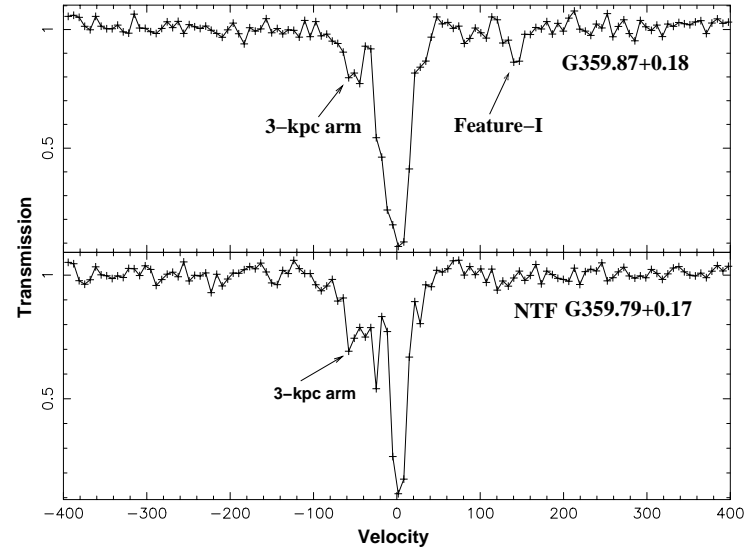

Fig. 7. HI absorption spectrum towards the extragalactic source G359.87+0.18 and the NTF G359.79+0.17. The bandwidth is $4 \mathrm{MHz}$. RMS noise in the two spectra are 0.028 and 0.026 respectively.

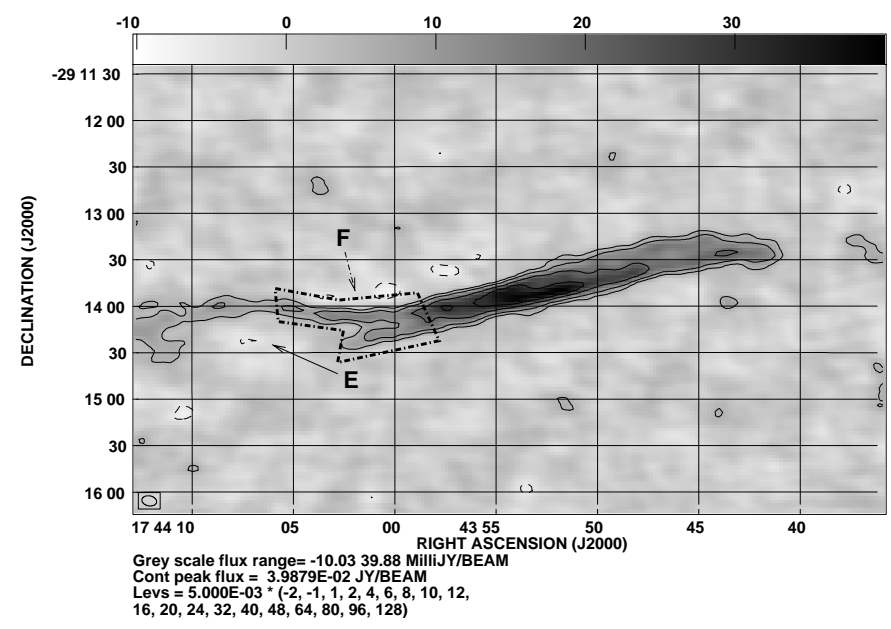

Fig. 8. Continuum image of G359.54+0.18 at $1.4 \mathrm{GHz}$ with a resolution of $9.6 \times 6.4 \operatorname{arcsec}^{2}$, along PA $=79^{\circ}$. The rms noise is $1.7 \mathrm{mJy}^{\text {beam }}{ }^{-1}$.

(Fig. 9) at this velocity ( $4 \sigma$ detection). Staguhn et al. (1998) also detected another molecular cloud near $-90 \mathrm{~km} \mathrm{~s}^{-1}$ near the eastern edge of the NTF (not seen in Fig. 8). Since the eastern edge of the NTF G359.54+0.18 is faint in radio-continuum, no useful $\mathrm{HI}$ absorption spectrum could be obtained.

\section{Discussion}

\subsection{Identification of $\mathrm{HI}$ features and constraints on the distances to the NTFs}

Identifications of HI absorption feature is performed by comparison with features of known velocities. Absorption indicates that the continuum source is located on the far side of the HI cloud and thereby provides a constraint on the distance to the continuum source. The velocities and the distances of the known HI emission features towards the three NTFs studied here have been discussed in Sect. 1 and summarised in Table 2, which will be used to constrain the distances to the NTFs. 
Table 2. Velocities and distances of the HI emission features.

\begin{tabular}{lccc}
\hline \hline $\begin{array}{l}\text { Name of the HI } \\
\text { feature }\end{array}$ & $\begin{array}{c}\text { Velocity } \\
\mathrm{km} \mathrm{s}^{-1}\end{array}$ & $\begin{array}{c}\text { Distance } \\
\mathrm{kpc}\end{array}$ & $\begin{array}{c}\text { References for } \\
\text { distances }\end{array}$ \\
\hline "0 km feature" & -30 to 30 & Unknown & \\
"3 kpc arm" & -53 & 5.1 & Cohen \& Davies (1976)* \\
"Molecular ring" & $\approx-135$ & $\lesssim 8.5$ & Cohen \& Davies (1979) \\
"Nuclear disk" & $\approx-170$ & $\lesssim 8.5$ & $"$ \\
"XVI" & +135 & $\approx 8.5$ & Cohen \& Davies (1976) \\
"I" & +135 & 10.5 & C
\end{tabular}

* Distance of " $3 \mathrm{kpc}$ arm” corrected for a Galactic Centre distance of $8.5 \mathrm{kpc}$ rather than $10 \mathrm{kpc}$.

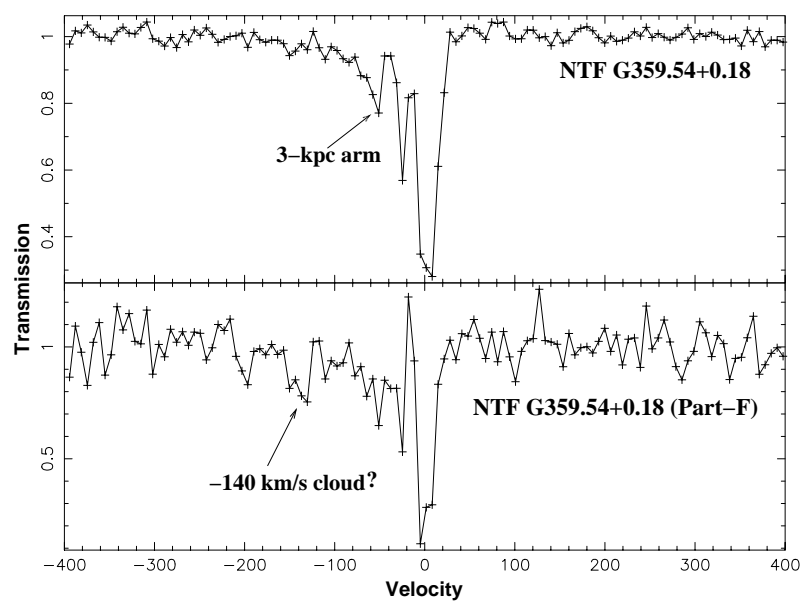

Fig. 9. HI absorption spectrum integrated over the NTF G359.54+0.18 and towards a smaller portion of the NTF where it bends (region "F" in Fig. 8). The bandwidth is $4 \mathrm{MHz}$. RMS noise in the two spectra are 0.025 and 0.1 respectively.

\subsubsection{Sgr C}

Due to absorption by the line of sight HI gas and velocity crowding near $l=0^{\circ}$, strong absorption is observed near $0 \mathrm{~km} \mathrm{~s}^{-1}$ in all spectra towards the Sgr C NTF and the HII region discussed in Sect. 3.1. Absorption by the " $3 \mathrm{kpc}$ arm" is observed at $-54 \mathrm{~km} \mathrm{~s}^{-1}$ towards the Sgr C NTF and the HII region (Fig. 3 and 4). However, the broad absorption feature (Fig. 3) identified towards the central part of the Sgr C NTF (marked "A" in Fig. 1) between -100 and $-200 \mathrm{~km} \mathrm{~s}^{-1}$ is peculiar. Three distinct absorption features are detected in the same velocity range in the higher resolution spectrum of the HII region in Fig. 4. This suggests absorption by clouds whose velocities differ by an amount less than the individual line-width.

Absorption near $-138 \mathrm{~km} \mathrm{~s}^{-1}$ is likely to be caused by the HI associated with the "Molecular ring", which has a linewidth of $\approx 40 \mathrm{~km} \mathrm{~s}^{-1}$ in emission (Cohen \& Davies 1979). Detection of absorption beyond $-160 \mathrm{~km} \mathrm{~s}^{-1}$ indicates absorption by the "Nuclear disk" (Rougoor \& Oort 1960). However, we were unable to identify the $-100 \mathrm{~km} \mathrm{~s}^{-1}$ absorption with any known HI emission feature. We believe that this feature was missed due to beam dilution in the low resolution (single dish) HI surveys. We examined the existing CO (Oka et al. 1998) and CS (Tsuboi et al. 1999) emission line maps of this region (spatial resolution $\sim 1^{\prime}$ ) made using the $45 \mathrm{~m}$ telescope of the Nobeyama Radio Observatory. Since these spectra have a much higher angular resolution than the single dish HI surveys, we attempted an identification of the $-100 \mathrm{~km} \mathrm{~s}^{-1}$ feature with a CO or CS feature. The CO spectra towards parts " $\mathrm{A}$ " and "B" of the NTF and the HII region is shown in Fig. 10. Strong CO emission is indeed observed near $-100 \mathrm{~km} \mathrm{~s}^{-1}$ (linewidth $\approx 20 \mathrm{~km} \mathrm{~s}^{-1}$ ). The antenna temperature from part " $\mathrm{A}$ " of the NTF is a factor of 2 higher than part " $\mathrm{B}$ ". The CS spectrum also shows a similar ratio. (CS emission traces dense molecular clouds with density $\sim 10^{4} \mathrm{~cm}^{-3}$, which are typically found in the CMZ). Figure 11 shows a contour image of this cloud in CS emission (data courtesy Tsuboi et al. 1999) within a velocity range of -110 and $-90 \mathrm{~km} \mathrm{~s}^{-1}$. Note that the molecular cloud covers almost the whole NTF, although the $-100 \mathrm{~km} \mathrm{~s}^{-1}$ absorption is only observed towards part " $\mathrm{A}$ ".

The $-100 \mathrm{~km} \mathrm{~s}^{-1}$ molecular cloud has been catalogued by Oka et al. (2001) and by Miyazaki \& Tsuboi (2000) (cloud 17 at $\left.l=359.48^{\circ}, b=-0.042^{\circ}\right)$. Using the estimated parameters of the cloud (radius $\approx 5 \mathrm{pc}$, mass $7.2 \times 10^{4} M_{\odot}$ ), the mean density of this cloud is $3100 \mathrm{H}_{2} \mathrm{~cm}^{-3}$. Assuming the ratio of atomic to molecular hydrogen is 0.01 (Liszt et al. 1983; Lasenby et al. 1989), the HI column density is $9.3 \times 10^{20} \mathrm{~cm}^{-2}$. We find that an assumed spin temperature of $100 \mathrm{~K}$, and a line width of $20 \mathrm{~km} \mathrm{~s}^{-1}$ explains the observed HI optical depth of $\approx 0.5$. Hence, we believe that we are observing absorption by $\mathrm{HI}$ associated with the $-100 \mathrm{~km} \mathrm{~s}^{-1}$ molecular cloud.

Our high resolution spectrum of the Sgr C HII region clearly shows the presence of two spectral features in the range of -50 to $-65 \mathrm{~km} \mathrm{~s}^{-1}$ (Fig. 4). Liszt \& Spiker (1995) have identified a molecular cloud towards Sgr C with a velocity of $\approx-65 \mathrm{~km} \mathrm{~s}^{-1}$, which they call M359.5-0.15. The $-54 \mathrm{~km} \mathrm{~s}^{-1}$ feature is identified with the $3 \mathrm{kpc}$ arm, whereas the $-61.5 \mathrm{~km} \mathrm{~s}^{-1}$ feature in our HI data is believed to arise from M359.5-0.15. The presence of absorption by the molecular cloud M359.5-0.15 indicates that the Sgr C HII region is either embedded in or located at the far side of the cloud. This result supports the suggestion of Liszt \& Spiker (1995) that the Sgr C HII region (Fig. 2) is located in a cavity of M359.5-0.15. We note that $\mathrm{CO}$ emission near $-65 \mathrm{~km} \mathrm{~s}^{-1}$ has been detected towards both part "A" of the NTF and the HII region (Fig. 10). The lack of HI absorption towards part "A" of the NTF indicates that it is located in the front of the $-65 \mathrm{~km} \mathrm{~s}^{-1}$ cloud. Therefore, we believe that the Sgr C HII region is relatively farther away than the Sgr C NTF, which provides evidence against any 


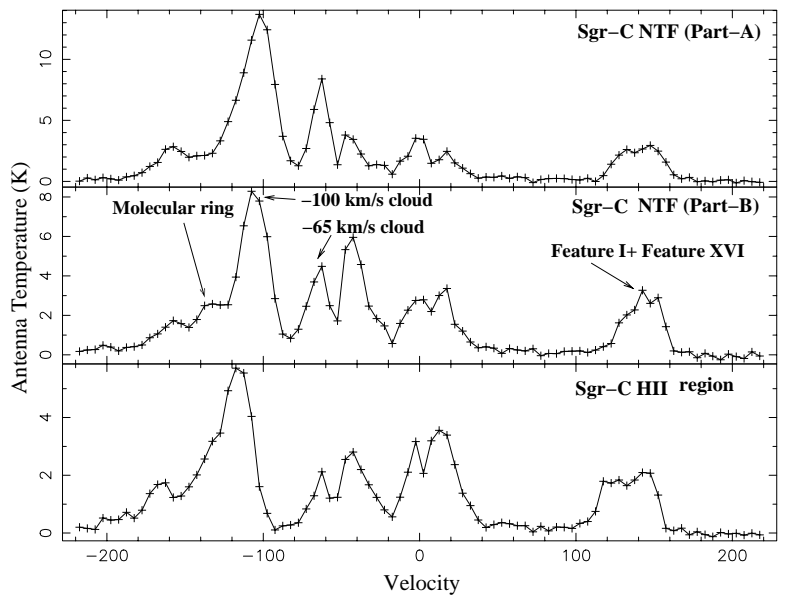

Fig. 10. CO emission spectra towards the central part (top panel) (marked "A" in Fig. 1) and the eastern part of the NTF (middle), along with the spectrum taken towards the Sgr C HII region (bottom) (Data courtesy Oka et al. 1998).

interaction between the two (see the schematic in Fig. 13). Having identified the absorption features in the HI spectrum, here we estimate the distance of the Sgr C complex. Since absorption by the "Molecular ring" and the "Nuclear disk" have been detected towards the Sgr C NTF (part "A" in Fig. 1) and the HII region, we can conclude that these objects seen in radio continuum are located at a minimum distance of these HI features. As the "Molecular ring" and the "Nuclear disk" are located within $\sim 200 \mathrm{pc}$ of the GC, this provides a lower limit of $\approx 8.3 \mathrm{kpc}$ to these objects. We note that despite the emission feature seen in the $\mathrm{CO}$ map at $\sim 140 \mathrm{~km} \mathrm{~s}^{-1}$ (Fig. 10), no corresponding $\mathrm{HI}$ absorption could be detected towards the Sgr $\mathrm{C}$ region. The $\mathrm{CO}$ emission from the molecular cloud at $\sim 140 \mathrm{~km} \mathrm{~s}^{-1}$ is likely to be associated with the HI feature "XVI" and "I" and both these features are located at the far side of the GC. Absence of any absorption by the HI associated with these structures indicate that the Sgr C NTF and the HII region are located within a few hundred parsecs at the far side of the GC, which provides an upper limit to their distances.

There appears to be weak absorption ( $4 \sigma$ detection) towards part "B" of the NTF at $-137 \mathrm{~km} \mathrm{~s}^{-1}$ which appears to have been caused by the "Molecular ring". Since we expect a much stronger absorption if this part of the NTF is located at the far side of the "Molecular ring", we suggest that the part "B" of the NTF is embedded in the "Molecular ring". Also, since the "Nuclear disk" is expected to be located farther away from the GC than the "Molecular ring"; lack of absorption by this feature may indicate that there is a hole in the "Nuclear disk" towards part "B" of the NTF.

\subsubsection{Objects located in the field of Sgr C: sources C, $\mathrm{D}$ and FIR-4}

The HI spectrum (Sect. 3.2) towards source "C" (Fig. 1) shows absorption at $-53 \mathrm{~km} \mathrm{~s}^{-1},-123 \mathrm{~km} \mathrm{~s}^{-1}$ and $-170 \mathrm{~km} \mathrm{~s}^{-1}$ (Fig. 5), which indicates HI absorption by the "3 kpc arm", "Molecular ring" ("Molecular ring" is expected to have a

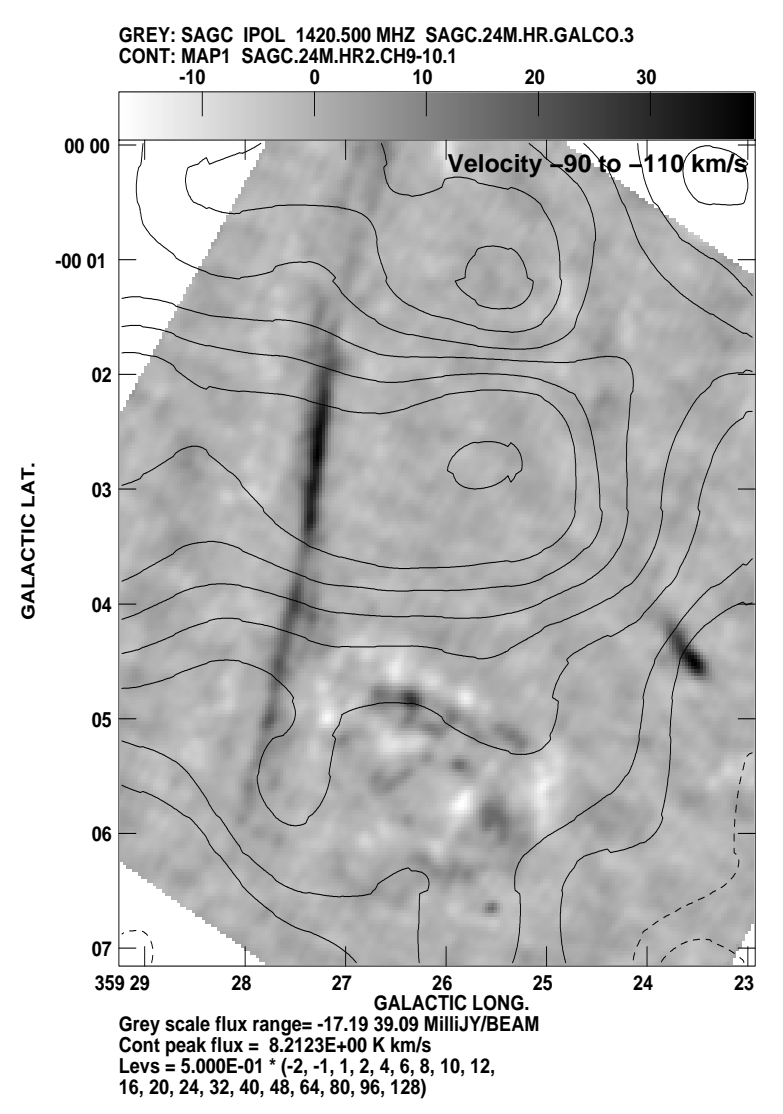

Fig. 11. CS emission (contours) averaged over a velocity range of -110 to $-90 \mathrm{~km} \mathrm{~s}^{-1}$ superimposed on a grey scale radio-continuum image of the Sgr C NTF in Galactic co-ordinate. CS data courtesy Tsuboi et al. (1999).

velocity of $\approx-135 \mathrm{~km} \mathrm{~s}^{-1}$, which differs in this case by less than the line-width) and the "Nuclear disk" respectively. HI spectrum of FIR-4 also indicates absorption by the "Molecular ring" at $-135 \mathrm{~km} \mathrm{~s}^{-1}$ and the "Nuclear disk" at $\approx-183 \mathrm{~km} \mathrm{~s}^{-1}$ (Fig. 5). Since the "Molecular ring" and the "Nuclear disk" are thought to be located within a few hundred parsecs of the GC, HI absorption by these two features provides a lower limit of $\approx 8.5 \mathrm{kpc}$ to the distances of the source "C" and FIR-4. On the other hand, the spectrum taken towards the southern HII region "D" (Fig. 2), shows no absorption near $-53 \mathrm{~km} \mathrm{~s}^{-1}$ (Fig. 5). Liszt \& Spiker (1995) have suggested that source "D" is a local object due to the absence of non-zero velocity features in the HI spectrum. The absence of any non-zero velocity features in our spectrum also makes us draw a similar conclusion.

\subsubsection{NTF G359.79+0.17 \& G359.87+0.18}

$\mathrm{HI}$ absorption near $+140 \mathrm{~km} \mathrm{~s}^{-1}$ is observed (Fig. 7) towards the extragalactic source G359.87+0.18. The HI emission feature "I" seen in HI (Cohen \& Davies 1979) is located $\approx 2 \mathrm{kpc}$ behind the GC and has a line of sight velocity of $+135 \mathrm{~km} \mathrm{~s}^{-1}$ at this longitude. The HI feature "XVI" located a few hundred parsecs behind the GC also has a similar velocity at this longitude. The absorption seen in Fig. 7 near $+140 \mathrm{~km} \mathrm{~s}^{-1}$ matches closely the velocity of these two features, which indicates that 


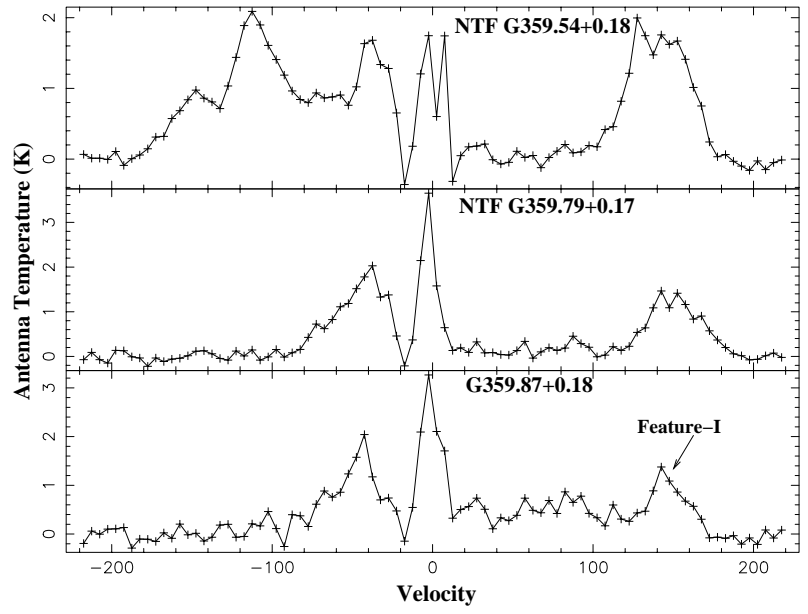

Fig. 12. CO emission spectrum towards the NTF G359.54+0.18 (top), G359.79+0.17 (middle) and the extragalactic source G359.87+0.18 (bottom). Data courtesy Oka et al. (1998).

the absorption is caused by either one or a combination of both these features.

No HI absorption at high positive velocity is detected towards the NTF G359.79+0.17. However, CO emission has been detected near $+140 \mathrm{~km} \mathrm{~s}^{-1}$ towards both $\mathrm{G} 359.79+0.17$ and the extragalactic source G359.87+0.18 (Fig. 12), which indicates that there is no hole in feature "I" (or perhaps in feature "XVI") along these directions. Consequently, the upper limit to the distance of the NTF is $\approx 10.5 \mathrm{kpc}$. The presence of absorption in the spectrum of the NTF up to a negative velocity of $-58 \mathrm{~km} \mathrm{~s}^{-1}$ suggests absorption by the " $3 \mathrm{kpc}$ arm" and consequently, the lower limit to its distance is $\approx 5.1 \mathrm{kpc}$ of the Sun.

Lazio et al. (1999) suggested the presence of a $-20 \mathrm{~km} \mathrm{~s}^{-1}$ cloud at the far side of the GC. The presence of a narrow absorption feature $-26 \mathrm{~km} \mathrm{~s}^{-1}$ in our data towards the NTF $\mathrm{G} 359.79+0.17$ may indicate that it is embedded in this negative velocity cloud. This cloud could have caused the somewhat wider absorption profile in the spectrum towards the extragalactic source G359.87+0.18 which is observed through a substantially longer line of sight path through this HI gas.

\subsubsection{NTF G359.54+0.18}

The spectrum of G359.54+0.18 (Fig. 9) is quite similar to that towards the NTF G359.79+0.17. The presence of absorption by the " $3 \mathrm{kpc}$ arm" near a velocity of $-53 \mathrm{~km} \mathrm{~s}^{-1}$ clearly indicates that G359.54+0.18 is located beyond $5.1 \mathrm{kpc}$ from the Sun. As G359.79+0.17 and G359.87+0.18, CO emission has also been detected towards this NTF at $140 \mathrm{~km} \mathrm{~s}^{-1}$ (Fig. 12), but, absence of $\mathrm{HI}$ absorption at this high positive velocity indicates that it is located within $\approx 10.5 \mathrm{kpc}$ from the Sun. As described in section Sect. 4.1.3, this NTF also seems to be embedded in the $-20 \mathrm{~km} \mathrm{~s}^{-1}$ cloud. We could detect weak HI absorption at $4 \sigma$ level at $-140 \mathrm{~km} \mathrm{~s}^{-1}$ (Fig. 9) toward part-F of the NTF shown in Fig. 8. A dense molecular cloud having the same velocity is also found to be present near this location (Staguhn et al. 1998). Since dense molecular cloud are typically found only in the CMZ, our observations suggest that the NTF G359.54+0.18 is also embedded in or located at the far side of the CMZ.

\subsection{HI column densities}

We have estimated column densities for the HI feature "I" $\left(+155\right.$ to $\left.+125 \mathrm{~km} \mathrm{~s}^{-1}\right)$, the local foreground HI component ( +35 to $\left.-35 \mathrm{~km} \mathrm{~s}^{-1}\right)$, the " $3 \mathrm{kpc}$ arm" $\left(-77\right.$ to $\left.-40 \mathrm{~km} \mathrm{~s}^{-1}\right)$, and the combined molecular cloud and "Nuclear Disk" ( -90 to $-200 \mathrm{~km} \mathrm{~s}^{-1}$ ) components towards the NTFs Sgr C, G359.79+0.17 and G359.54+0.18 and the extragalactic source G359.87+0.18. These column densities, expressed as a multiple of the spin temperature " $T_{\mathrm{s}}$ ", are presented in Table 3. The velocity limits for the various $\mathrm{HI}$ features were determined by visual inspection of the absorption spectra towards these sources. Since we could not separate the absorption by the molecular cloud and the "Nuclear disk", we have quoted their total column densities. Due to the velocity crowding towards the GC, the optical depth of the local line of sight HI gas is large (i.e., $\exp (-\tau) \approx 0$ ). Therefore, any small error in estimating the optical depth would result in a comparatively larger error in the estimated column densities. Hence, the estimated total column densities of the foreground HI gas should be taken with caution. The column density of the line of sight HI gas $\left(-35 \mathrm{~km} \mathrm{~s}^{-1}<\right.$ velocity $<35 \mathrm{~km} \mathrm{~s}^{-1}$ ) shown in Table 3 falls by a factor of 2 towards the NTFs G359.79+0.17 and G359.54+0.18 for $|b|$ $\approx 0.2^{\circ}$, which suggests that the scale height of the $\mathrm{HI}$ gas at the distance of the GC is $\sim 27 \mathrm{pc}$.

The HI column density estimated from the optical depth towards the "Snake" (G359.1-0.2) NTF (Uchida et al. 1992) is $1.1 T_{\mathrm{s}} \times 10^{20}$ due to the local foreground HI component, $2.3 T_{\mathrm{s}} \times 10^{19}$ due to the " $3 \mathrm{kpc}$ arm", and $2.8 T_{\mathrm{s}} \times 10^{19}$ due to the "Molecular ring". These numbers are comparable (within a factor of two) with our estimated column density towards G359.54+0.18 and G359.79+0.17 given in Table 3. We note that the column density as quoted in Table 1 of Uchida et al. (1992) towards all of their sources are incorrect and 100 times higher than what is found from their quoted optical depth.

\subsection{Interaction of the molecular cloud with the Sgr C NTF}

As shown in Sect. 3.1, no HI absorption by the $-100 \mathrm{~km} \mathrm{~s}^{-1}$ cloud could be identified towards part "B" of the Sgr C NTF (Fig. 3). However, HI absorption by the $-100 \mathrm{~km} \mathrm{~s}^{-1}$ cloud could be identified towards part "A" of the Sgr C NTF. From the ratio of $\mathrm{CO}$ brightness and temperature towards part "A" and "B" of the NTF (Sect. 4.1.1), we expect HI optical depth of $\approx 0.2$ towards " $B$ " in the velocity range of -95 and $-125 \mathrm{~km} \mathrm{~s}^{-1}$, which is 5 times higher than the effective noise in the spectrum. Therefore, if the abundance of atomic hydrogen and the spin temperature $\left(T_{\mathrm{S}}\right)$ are the same in different parts of the cloud, HI absorption towards part "A" and the lack of it towards part " $\mathrm{B}$ " of the NTF can only be explained if " $\mathrm{A}$ " is located at the far side and " $\mathrm{B}$ " is located at the near side of the cloud and the NTF is at least partly embedded within the cloud. In Fig. 13, we show a schematic of the Sgr C complex in 
Table 3. HI column densities toward Sgr C, G359.79+0.17, G359.54+0.18 and G359.87+0.18.

\begin{tabular}{lccccc}
\hline \hline $\begin{array}{l}\text { Column density } \\
\left(\mathrm{cm}^{-2}\right)\end{array}$ & $\begin{array}{c}\text { Central part of } \\
\text { Sgr C NTF }\end{array}$ & $\begin{array}{c}\text { Sgr C HII } \\
\text { region }\end{array}$ & G359.79+0.17 & G359.54+0.18 & G359.87+0.18 \\
\hline$N(\mathrm{H})\left(+155\right.$ to $\left.+125 \mathrm{~km} \mathrm{~s}^{-1}\right)$ & - & - & - & - & $5.2 \times 10^{18} T_{\mathrm{s}}$ \\
$N(\mathrm{H})\left(+35\right.$ to $\left.-35 \mathrm{~km} \mathrm{~s}^{-1}\right)$ & $1.6 \times 10^{20} T_{\mathrm{s}}$ & $2.3 \times 10^{20} T_{\mathrm{s}}$ & $8.8 \times 10^{19} T_{\mathrm{s}}$ & $6.3 \times 10^{19} T_{\mathrm{s}}$ & $1.3 \times 10^{20} T_{\mathrm{s}}$ \\
$N(\mathrm{H})\left(-77\right.$ to $\left.-40 \mathrm{~km} \mathrm{~s}^{-1}\right)$ & $3.0 \times 10^{19} T_{\mathrm{s}}$ & $2.7 \times 10^{19} T_{\mathrm{s}}$ & $1.8 \times 10^{19} T_{\mathrm{s}}$ & $1.0 \times 10^{19} T_{\mathrm{s}}$ & $1.9 \times 10^{19} T_{\mathrm{s}}$ \\
$N(\mathrm{H})\left(-90\right.$ to $\left.-200 \mathrm{~km} \mathrm{~s}^{-1}\right)$ & $4.8 \times 10^{19} T_{\mathrm{s}}$ & $3.6 \times 10^{19} T_{\mathrm{s}}$ & - & - & - \\
\hline
\end{tabular}

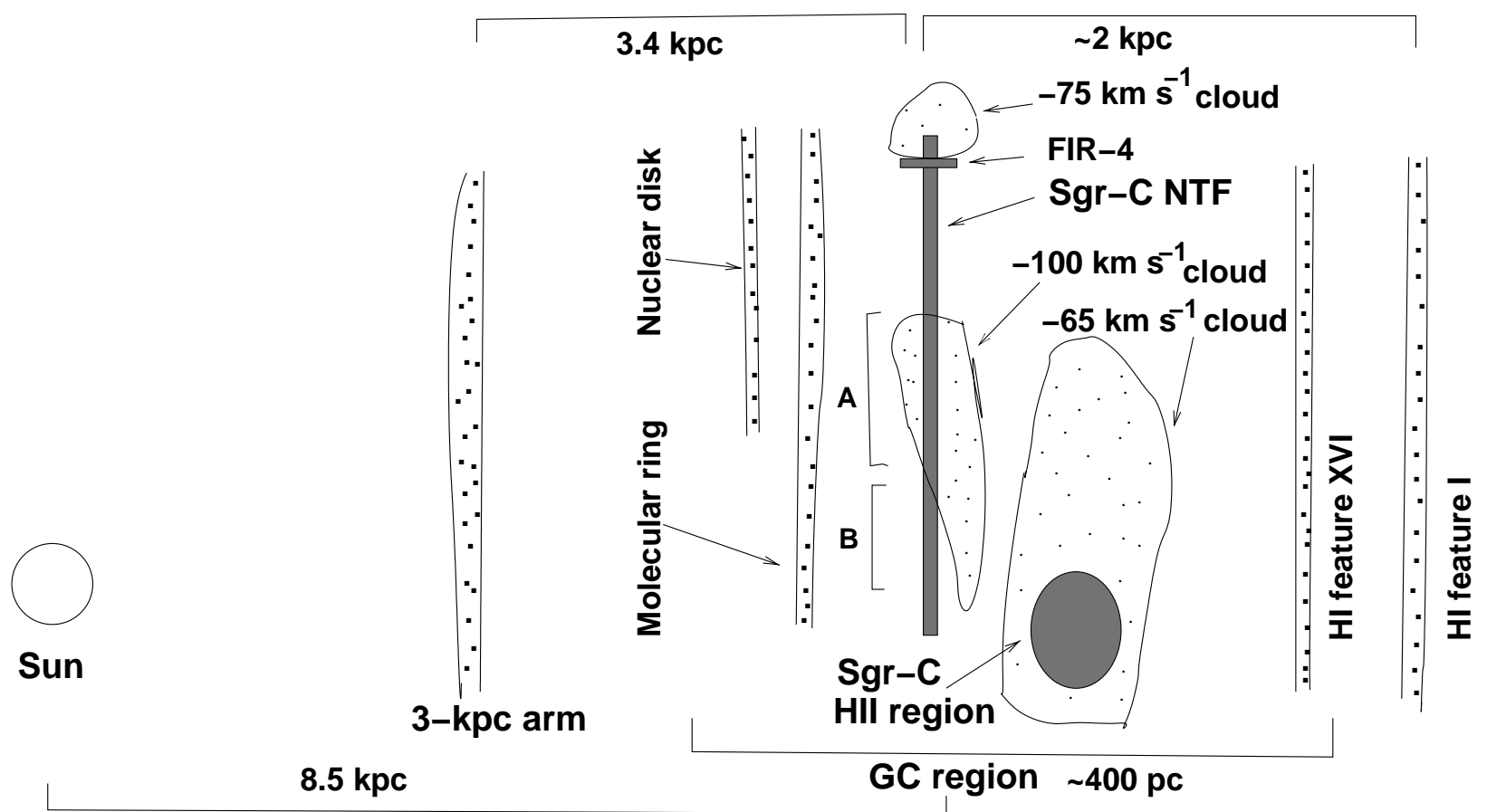

Fig. 13. Schematic diagram of the Sgr C complex with HI absorbing clouds (not to scale) as seen from bottom of the Galaxy.

the $\mathrm{GC}$ region, including the relative location of the absorbing clouds about the Sgr C complex.

Here, we consider three possible scenarios that could have led the Sgr C NTF to be partly embedded within the $-100 \mathrm{~km} \mathrm{~s}^{-1}$ cloud.

(i) We first consider the case of the NTF tunnelling through the already existing $-100 \mathrm{~km} \mathrm{~s}^{-1}$ cloud after being created. To penetrate the cloud, the high energy electrons in the NTF have to first ionise the neutral gas. With an estimated width of $35^{\prime \prime}$ $(1.4 \mathrm{pc})$, length $255^{\prime \prime}(10 \mathrm{pc})$ and equipartition magnetic field of $100 \mu \mathrm{G}$ (Anantharamaiah et al. 1991) in the NTF, the estimated total energy of the relativistic electrons is $\sim 2 \times 10^{47} \mathrm{erg}$. However, the energy required to ionise even a HI cloud (mean density $3100 \mathrm{~cm}^{-3}$ ) of length equal to that of the size of the cloud $(10 \mathrm{pc})$ and cross-sectional area equal to that of the NTF is $\sim 3 \times 10^{49} \mathrm{erg}$. Therefore, the energy of the relativistic electrons is less than $1 \%$ of the energy required to penetrate the molecular cloud. Hence, unless a tunnel in the cloud already exists, the electrons will not have sufficient energy to support this scenario.

(ii) Next, we consider the case, where the NTF does not penetrate the cloud, but the synchrotron electrons are generated from the ionised surfaces on either side of the $-100 \mathrm{~km} \mathrm{~s}^{-1}$ cloud and follow the local magnetic field lines. In the case of the "Radio-arc", which is comprised of several narrow filaments, the individual filaments appear to either end or begin from the HII region G0.18-0.04 (sickle like feature), located near the midpoint of the NTF. Based on the observations of the "Radio-arc", Serabyn \& Morris (1994) have proposed that association of the NTF, molecular cloud and HII region are necessary to explain the generation of high energy electrons forming the NTFs. In Fig. 2c of Liszt \& Spiker (1995), we observe two filamentary structures towards the western side of the part "A" of Sgr C NTF, which merge into a single brighter filament near "A". Our observations indicate that the $-100 \mathrm{~km} \mathrm{~s}^{-1}$ cloud is associated with the NTF near "A" $\left(\mathrm{RA}(\mathrm{J} 2000)=17^{\mathrm{h}} 44^{\mathrm{m}} 33^{\mathrm{s}}\right.$, Dec $\left.=-29^{\circ} 25^{\prime} 55^{\prime \prime}\right)$. In this scenario, if the surface of this cloud is ionised, then association of the molecular cloud and HII region with the NTF satisfies the criterion of Serabyn \& Morris (1994) to generate it. Here, if the electrons flowing towards part " $A$ " is at the far side and part " $\mathrm{B}$ " lies at the near side of the cloud, then the absence of $-100 \mathrm{~km} \mathrm{~s}^{-1} \mathrm{HI}$ absorption against part "B" can be explained. However, no such ionised surface of the cloud (as HII region) 
like G0.18-0.04 has been detected near part "A" of the NTF from the Nobeyama millimetre array observation of this region (Tsuboi et al. 1991) at $22 \mathrm{GHz}$. Thus, the above scenario also fails to explain the observations.

(iii) Finally, we consider an alternative source of the synchrotron electrons in the Sgr C NTF and the case of collision of the $-100 \mathrm{~km} \mathrm{~s}^{-1}$ cloud with this already existing NTF. Liszt \& Spiker (1995) proposed that the HII region FIR 4 (Odenwald \& Fazio 1984) is associated with the Sgr C NTF, which acts as the source of relativistic electrons. Figure 7 in Liszt \& Spiker (1995) shows a molecular cloud around this HII region. We note that FIR-4, with its wedge-like morphology, is similar to the HII region G0.18-0.04, discussed earlier. The longer side of FIR-4 lies almost perpendicular to the direction of the NTF, thereby suggesting interaction between the NTF and the HII region in a way similar to the Radio-arc. The CS data (Tsuboi et al. 1999) shows a compact dense molecular cloud with a velocity of $-75 \mathrm{~km} \mathrm{~s}^{-1}$, which coincides with the position of FIR-4. However, in the absence of any recombination line detection from this HII region, actual association of FIR-4 and the $-75 \mathrm{~km} \mathrm{~s}^{-1}$ cloud cannot be verified. In Sect. 4.1.2, it is shown that the lower limit to its distance is $\approx 8.5 \mathrm{kpc}$, which is consistent with its proposed association with the NTF. Therefore, if we assume that the FIR-4 acts as the source of relativistic electrons to the Sgr C NTF, collision of the $-100 \mathrm{~km} \mathrm{~s}^{-1}$ cloud with the central part of the NTF, which causes the NTF to appear partly embedded in the cloud, can also explain the observations. After the collision of the cloud with the NTF, if the magnetic pressure in the NTF would have been less than that of the cloud, the fields could be pinched or disrupted and the flow of high energy electrons towards part "B" significantly reduced. On the other hand, if the magnetic pressure in the NTF is much larger than the pressure due to the cloud (Yusef-Zadeh \& Morris 1987), then the NTF will remain stable and the cloud may wrap around the NTF. Here, the flow of electrons from the FIR-4 to part "B" of the NTF will not be significantly disrupted.

If flow of electrons has stopped in part "B" (first possibility), then this part of the NTF is likely to show a steeper spectral index. However, the estimated spectral index between 1.6 GHz and $330 \mathrm{MHz}$ is quite flat, which LaRosa et al. (2000) has suggested to be due to the presence of thermal emission from the nearby HII region. In this regard, we note that if the $-100 \mathrm{~km} \mathrm{~s}^{-1}$ cloud is moving with a velocity similar to its line of sight velocity in the sky plane, then the collision took place around $5 \times 10^{4}$ years ago. However, to our knowledge, part "B" of the NTF has been imaged with high resolution and sensitivity only up to a few $\mathrm{GHz}$ and at this frequency range, the half-life of synchrotron electrons responsible for the emission in an equipartition field of $0.1 \mathrm{mG}$ (LaRosa et al. 2000) is $\sim 10^{5}$ years. Therefore, unless the magnetic field is much higher than the equipartition value, no significant steepening of the spectral index may be detectable in the presently available data. Hence, to distinguish between the two cases, future polarimetric observations, which traces the direction of the local magnetic field lines, and can show that whether the magnetic field lines in part "B" are pinched, will be useful. We prefer the third model, taking into consideration the available information. Since the NTF does not show any change of shape or direction near the place of interaction with the $-100 \mathrm{~km} \mathrm{~s}^{-1}$ cloud, the magnetic pressure in the NTF is likely to be more than the pressure of the cloud.

\section{Conclusions}

HI absorption studies of three NTFs known as the Sgr C, G359.54+0.18 and G359.79+0.17 using the GMRT have yielded the following results:

(a) For the first time, the Sgr C NTF and the HII region are shown to be located within a few hundred parsecs of the GC.

(b) Our study indicates that the Sgr C HII region is either embedded in or located behind the $-65 \mathrm{~km} \mathrm{~s}^{-1}$ molecular cloud, whereas the Sgr C NTF is located at the near side of the cloud, which argues against any possible interaction between the two objects.

(c) A molecular cloud with a velocity of $-100 \mathrm{~km} \mathrm{~s}^{-1}$ appears to be associated with the central part of the Sgr C NTF, and on the basis of the presently existing data, it appears that the magnetic pressure in the NTF is higher than the pressure due to the $-100 \mathrm{~km} \mathrm{~s}^{-1}$ cloud.

(d) HI absorption by the " $3 \mathrm{kpc}$ arm" is detected against all the three NTFs, which indicates that the NTF G359.54+0.18 and $\mathrm{G} 359.79+0.17$ are located at a minimum distance of $5.1 \mathrm{kpc}$ from the Sun.

(e) Weak HI absorption ( $4 \sigma$ level) at $-140 \mathrm{~km} \mathrm{~s}^{-1}$ suggests that the NTF G359.54+0.18 is located at a minimum distance of $\approx 8.5 \mathrm{kpc}$ from us.

(f) The maximum distance of the NTF G359.54+0.18 and $\mathrm{G} 359.79+0.17$ is estimated to be $10.5 \mathrm{kpc}$ from the Sun.

The present study extends the number of NTFs that have been found to be located near the GC region to five. With most of the known NTFs now being shown to be near the GC, there remains little doubt that phenomena related to the central region of the Galaxy are responsible for the creation and maintenance of the NTFs.

Acknowledgements. It is a pleasure to thank A. Pramesh Rao, with whom I have discussed several aspects of this work at various stages. I also thank Jayaram Chengalur, Miller Goss, Rajaram Nityananda, Dharam Vir Lal and Nimisha Kantharia for reading the manuscript and for their useful comments. Cornelia Lang, as the referee, has made several useful comments, which helped to improve the paper, and I would like to thank her. Masato Tsuboi and Tomoharu Oka provided their CS and CO survey data respectively and I wish to thank them. I thank the staff of the GMRT that made these observations possible. GMRT is run by the National Centre for Radio Astrophysics of the Tata Institute of Fundamental Research. I acknowledge of receiving partial funding from the Rekhi Scholarship of the TIFR Endowment Fund.

\section{References}

Anantharamaiah, K. R., Pedlar, A., Ekers, R. D., \& Goss, W. M. 1991, MNRAS, 249, 262

Bally, J., \& Yusef-Zadeh, F. 1989, ApJ, 336, 173

Cohen, R. J. 1975, MNRAS, 171, 659

Cohen, R. J., \& Davies, R. D. 1976, MNRAS, 175, 1

Cohen, R. J., \& Davies, R. D. 1979, MNRAS, 186, 453

Dickey, J. M., Terzian, Y., \& Salpeter, E. E. 1978, ApJS, 36, 77 
Kramer, C., Staguhn, J., Ungerechts, H., \& Sievers, A. 1998, in The Central Regions of the Galaxy and Galaxies, IAU Symp., 184, 173 Lang, C. C., Anantharamaiah, K. R., Kassim, N. E., \& Lazio, T. J. W. 1999, ApJ, 521, L41

LaRosa, T. N., Kassim, N. E., Lazio, T. J. W., \& Hyman, S. D. 2000, AJ, 119, 207

Lasenby, J., Lasenby, A. N., \& Yusef-Zadeh, F. 1989, ApJ, 343, 177

Lazio, T. J. W., Anantharamaiah, K. R., Goss, W. M., Kassim, N. E., \& Cordes, J. M. 1999, ApJ, 515, 196

Liszt, H. S., \& Spiker, R. W. 1995, ApJS, 98, 259

Liszt, H. S., van der Hulst, J. M., Burton, W. B., \& Ondrechen, M. P. 1983, A\&A, 126, 341

Miyazaki, A., \& Tsuboi, M. 2000, ApJ, 536, 357

Morris, M., \& Serabyn, E. 1996, ARA\&A, 34, 645

Odenwald, S. F., \& Fazio, G. G. 1984, ApJ, 283, 601

Oka, T., Hasegawa, T., Sato, F., Tsuboi, M., \& Miyazaki, A. 1998, ApJS, 118, 455
Oka, T., Hasegawa, T., Sato, F., et al. 2001, ApJ, 562, 348

Rougoor, G. W. 1964, Bull. Astron. Inst. Netherlands, 17, 381

Rougoor, G. W., \& Oort, J. H. 1960, Proc. Nat. Acad. Sci., 46, 1 Scoville, N. Z. 1972, ApJ, 175, L127

Serabyn, E., \& Morris, M. 1994, ApJ, 424, L91

Staguhn, J., Stutzki, J., Uchida, K. I., \& Yusef-Zadeh, F. 1998, A\&A, 336, 290

Swarup, G., Ananthakrishnan, S., Kapahi, V. K., et al. 1991, Curr. Sci., 60,95

Tsuboi, M., Handa, T., \& Ukita, N. 1999, ApJS, 120, 1

Tsuboi, M., Kobayashi, H., Ishiguro, M., \& Murata, Y. 1991, PASJ, 43, L27

Uchida, K., Morris, M., \& Yusef-Zadeh, F. 1992, AJ, 104, 1533

Uchida, K. I., \& Guesten, R. 1995, A\&A, 298, 473

Yusef-Zadeh, F., \& Morris, M. 1987, ApJ, 322, 721

Yusef-Zadeh, F., Wardle, M., \& Parastaran, P. 1997, ApJ, 475, L119 